\title{
QUAND LE CANCER RENCONTRE LE GENRE ${ }^{1}$
}

\section{Anastasia Meidani $^{2}$ - Alessandrin Arnaud ${ }^{3}$}

Résumé : Cet article revient sur le volet français et qualitatif d'une recherche européenne de méthodologie mixte portant sur les expériences du cancer. À travers 100 entretiens auprès de malades, leurs proches et les professionnel·le·s impliquée.es dans leur prise en charge et 400 observations in situ réalisées dans cinq établissements de soins, il explore la manière dont le genre intervient dans le travail du malade et les expériences de soins. L'analyse se déploie en trois temps. Dans une première partie, la reproduction des normes de genre est interrogée à partir d'un double paradoxe : la «vulnérabilité » apparente des femmes se couple à une résistance sociale et relationnelle forte, là où l'endurance supposée des hommes laisse souvent place à des subjectivités affaiblies. Dans une seconde partie, les ajustements de genre que la maladie initie sont discutés afin de distinguer ce qui, en termes de masculinités et de féminités plurielles, contribue à la mise en place des stratégies visant à tenir tête à la maladie. Enfin, dans une troisième partie, les relations de soins sont scrutées à partir de ce double mouvement qui oscille entre reproduction normative et ajustements de genre situés.

Mots clés : genre, cancer, masculinités, féminités.

Summary: This article reviews the French and qualitative aspects of a mixed-method European research on cancer experiences. Through 100 interviews with patients, their ones

\footnotetext{
${ }^{1}$ Pour citer cet article : Meidani, Anastasia, et Arnaud Alessandrin. "Quand le cancer rencontre le genre ", Revue française de sociologie, vol. 60, no. 2, 2019, pp. 201-224.

${ }^{2}$ Maitresse de conférence à l'Université Toulouse Jean Jaures, LISST. Auteure de « Parcours de santé / Parcours de genre " (codir. avec A. Alessandrin), PUM, 2018 ; "La santé : du public à l'intime » (codir. avec E. Legrand et B. Jacques), EHESP, 2015 ; « Les fabriques du corps », PUM, 2007.

${ }^{3}$ Sociologue, Chercheur associé au LACES (Université de Bordeaux). Auteur de "Actualité des trans studies » (dir.) EAC, 2019 ; "Sociologie des transidentités », Cavalier Bleu, 2018 ; " Genre : l'essentiel pour comprendre » (avec B. Esteve-Bellebeau), DASUT, 2014.
} 
and the professionals involved in their care and 400 in-situ observations made in five health care facilities, it explores how gender intervenes in the work of the patient and the experiences of care. The analysis unfolds in three stages. In the first part, the reproduction of gender norms is questioned from a double paradox: the apparent "vulnerability" of women is coupled with strong social and relational resistance, where the supposed endurance of men often gives way to weakened subjectivities. In a second part, the gender adjustments that the disease initiates are discussed in order to distinguish what, in terms of plural masculinities and feminities, contributes to the implementation of strategies to cope with the disease. Finally, in a third part, the interactions of care are scrutinized from this double movement that oscillates between normative reproduction and situated gender adjustments.

Key words: gender, cancer, masculinities, feminities. 


\section{Introduction $^{4}$}

En 2017, le nombre de nouveaux cas de cancers en France métropolitaine est estimé à 400 000, dont 214000 chez l'homme, alors que la même année, près de 150000 décès par cancer sont répertoriés dont un peu plus de 84000 concernent les hommes ( $\mathrm{INCa}^{5}$ ). En même temps les cancers progressent jusqu'à devenir la première cause de mortalité ${ }^{6}$, avec le cancer du sein situé toujours en tête chez les femmes et suivi de près par le cancer du poumon - première cause de mortalité chez les hommes. Quant à l'évolution de la répartition sexuée des statistiques de mortalité par cancer, elle interroge. À titre d'exemple et pour le cancer du poumon, elle atteste d'une baisse de la mortalité chez la population masculine, divisée par 2 en 10 ans, et d'une augmentation conséquente chez la population féminine, multipliée par 4 en 15 ans. Cette dernière laisse présager d'une réduction graduelle de l'avantage relatif enregistré pour les femmes en termes d'espérance de vie (Meslé, 2004), et une recrudescence des inégalités sociales de santé au sein même de la population féminine, comme cela s'observe déjà pour la mortalité prématurée depuis la fin des années 1960 (Menvielle et al., 2007).

La sociologie française est restée étonnement silencieuse face au panorama épidémiologique de ces écarts sexués dont le présent article se propose de rendre compte, prenant appui sur le volet français et qualitatif d'une enquête européenne de 3 ans, de méthodologie mixte, soutenue par l'INCa et réalisée entre 2015 et 2018. En matière d'analyse, si les cas infirmatoires - émanant surtout des cancers «mixtes»: tumeurs du poumon et du côlonrectum - ont été pris en compte afin d'appréhender la complexité des phénomènes étudiés,

\footnotetext{
${ }^{4}$ Pour cet article nous avons opté pour une écriture inclusive par usage d'un point médian et avons suivi les préconisations du Haut Conseil à l'Egalité entre les Femmes et les Homme en matière de communication sans stéréotype de sexe (HCEFH, 2015).

5 L'Institut National du Cancer https://www.e-cancer.fr/Professionnels-de-sante/Les-chiffres-du-cancer-enFrance/Epidemiologie-des-cancers/Donnees-globales

${ }^{6} \mathrm{http}: / /$ ec.europa.eu/eurostat/statistics-

explained/index.php/File:Causes_of_death_\%E2\%80\%94_standardised_death_rate,_2014_(per_100_000_inhabi tants)_YB17.png
} 
c'est la focalisation sur les cancers sexués qui a servi de point de départ au développement théorique. Cet effet de loupe a permis de mettre en exergue, et d'une certaine manière d'isoler, les aspects de l'expérience cancéreuse spécifiquement liés au genre, et c'est à ce titre qu'il est adopté dans le cadre du présent article qui braque le projecteur sur les cancers du sein et de la prostate.

\section{Problématique}

Une revue de littérature socio-anthropologique, pour l'essentiel anglophone, qui interroge le couple genre / santé fait valoir que les rapports sociaux de sexe jouent un rôle majeur dans la structuration des parcours de soins et la gestion des trajectoires de la maladie chronique (Ahmed et Bates, 2007 ; Abel et Nelson, 1990 ; Aronson, 1992 ; Duffy, 2005 ; Gerstel et Gallagher, 2001 ; Courtenay, 2000 ; O’brien, Hunt et Hart, 2007). Selon ces travaux, les corps des femmes sont placés sous l'examen minutieux de la médicine. De nombreux thèmes le relatent : la reproduction, l'accouchement, la ménopause, la menstruation, la sexualité, le poids médicalement recommandé, la large médicalisation des problèmes esthétiques... Dans cette perspective, l'histoire sociale de la gestion chirurgicale du cancer de sein illustre un mécanisme significatif de ce contrôle médical exercé sur les corps féminins (Potts, 2000), soumis à la conformité normative d'un système patriarcal. D'autres études montrent que les femmes qui assument des responsabilités familiales au quotidien tendent à se rapprocher des attentes culturelles sexo-spécifiques, ce qui influence la gestion de la maladie chronique (O’grady, 2005 ; McMahon, 1995). Par exemple, Cancian et Oliker (2000) rendent compte de la surreprésentation des femmes dans le travail de soins au sein de la sphère privée, le care, dont les caractéristiques ont été assimilées à des attributs féminins. D'autres études encore (Lorber et Moore, 2002 ; Meidani, 2007 ; 2008 ; Christias, 2009) montrent que les femmes apprennent à surveiller leur santé et la santé des autres, alors que la socialisation des hommes 
les incite à ne pas prêter attention à leur corps. Par ailleurs, les femmes utilisent le système de soins plus régulièrement que les hommes, ce qui leur vaut une certaine expérience du monde médical (Nussbaum, 2001), et sont plus enclines à reconnaître le caractère pathologique des symptômes, ce qui signifie qu'elles sont également plus disposées que les hommes à chercher et obtenir de l'aide (O’Grady, 2005).

En complément de ces travaux, la littérature - bien plus éparse - qui porte principalement sur les rapports que les hommes entretiennent avec la santé, s'attarde surtout sur le déni de la maladie chronique qui sape la capacité des malades à transmettre leurs besoins à leurs proches et les professionnel-le·s de soins, et entrave la mise en place des parcours de soins appropriés (O’Brien, Hunt et Hart, 2007 ; Courtenay, 2000 ; Connell, 2014 ; Meidani, 2018). D’après ces enquêtes, les appréhensions à se présenter comme un homme sans défense ou fragile, les difficultés à mettre des mots sur ses émotions, l'inquiétude d'être à la merci des soins octroyés essentiellement par des femmes, l'impossibilité d'exécuter certaines tâches et le sentiment d'inutilité suite à l'avènement de la maladie, ou encore la crainte d'une « insuffisance » sur le plan sexuel à cause des traitements, hantent les réactions de la majorité de la population masculine. Les hommes qui adhérent à une certaine image de la virilité semblent donc enfermés dans un noyau représentationnel dur - qui se déploie autour du triptyque : travail, sexualité, force -, préjudiciable pour le traitement de la maladie (Courtenay, 2000).

Par ailleurs, force est de constater que la plupart des recherches qui portent sur la santé, ou la maladie, et le genre s'attardent sur les femmes ou adoptent un regard bi-catégoriel, et souvent essentialisant, où le masculin et le féminin sont réduits à un sexe assigné à la naissance, esquissant des identités sexuées supposées homogènes (Courtenay, 2000 ; Connell, 2014). À 
contre-pied de ces études, nous soutenons que les féminités et masculinités sont plurielles et que leur analyse concomitante est requise pour restituer toute la complexité des expériences cancéreuses. En complétant les travaux de Connell (2014), nous considérons que non seulement le spectre des masculinités se compose de plusieurs figures - hégémonique, complices, subordonnées ou marginalisées -, mais que le caractère pluriel qui structure le masculin prend aussi place dans l'élaboration des féminités protestataires, conventionnelles ou ambivalentes (Meidani, 2018). Plus encore, nous avançons que l'analyse de la maladie chronique, saisie par les concepts de la sociologie du genre, permet une approche plus fine des expériences cancéreuses, appréhendée ici à travers cette question qui compose le cœur de notre article : comment le genre intervient dans l'histoire de la maladie des personnes singulières, et ceci à deux niveaux. Premièrement, en examinant la façon dont il se déploie dans le travail du malade (Carricaburu et Ménoret, 2004). Deuxièmement, en questionnant la manière dont le genre concourt à la construction sociale des expériences de soins, et notamment des interactions soignant·e·s / soigné·e·s.

La question de l'expérience est donc centrale dans notre analyse. Nous faisons le double constat que l'expérience cancéreuse est faite d'interactions successives, et portée par plusieurs rationalités d'action. Ces dernières se déclinent différemment selon la socialisation de genre, les stratégies mises en place par les malades pour contrer l'épreuve de la maladie mais aussi les logiques de subjectivation que les patient·e-s mobilisent (Dubet, 1995). Si la socialisation de genre renvoie à la mise en œuvre des normes de masculinité et de féminité, les pratiques de soins ne sont pas réductibles à de pures applications de codes intériorisés ou à des enchaînements de choix stratégiques. Les logiques de subjectivation de genre interviennent aussi dans cette entreprise, chaque patient·e essayant de «se vivre » comme maitre de son histoire. 
À travers ce travail, qui tantôt prend le pli des modèles de genre dominants tantôt les garde à distance, le malade cherche à donner du sens à son expérience de la maladie, s'interroge sur ses causes et essaie de gérer les incertitudes biographiques qui en découlent (Mathieson et Stam, 1995). Si dans la majorité des cas la survenue d'un cancer marque un avant et un après, il appelle aussi à un travail de reconstruction identitaire qui prône la continuité et implique le genre. Dès lors, l'analyse permet de discuter la notion de rupture biographique (Bury, 1982), et montre qu'elle n'est pas automatiquement reliée à l'avènement de la maladie.

En cherchant à rendre compte de la trajectoire cancéreuse, la littérature socio-anthropologique francophone s'est longtemps intéressée aux représentations de la maladie, aux temporalités engendrées par celle-ci ou encore aux logiques profanes et expertes qui l'animent, aux pratiques dites à risques - et en particulier à l'alcoolo-tabagisme -, et dans une moindre mesure aux risques professionnels et environnementaux, laissant largement en retrait les interactions de soins (Debrez et Rollin, 2016 ; Cohen et al., 2015 ; Bataille, 2003 ; Ménoret, 1999 ; Saillant, 1988, Thébaud-Mony, 2008 ; Legrand et Meidani, 2018 ; Herzlich et Pierret, 1984 ; Tarantini, Gallardo et Peretti-Watel, 2014). Dans le prolongement de ces travaux, nous nous proposons d'étudier l'expérience cancéreuse et l'expérience de soins en intégrant les questions du genre, appréhendé à travers une approche dynamique, contextuelle, relationnelle et intersectionnelle (Mccall, 2005 ; Bauer, 2014 ; Jaunait et Chauvin, 2013).

Si la lecture socio-anthropologique du cancer a déjà approché la question du genre en interrogeant les oppositions chiffrées et les stratégies différenciées mises en place par des femmes et des hommes pour faire face à la maladie (Meidani, 2007 ; 2008 ; 2018 ; Meidani et Garnung, 2009 ; Jacques, 2009 ; Tarantini, Gallardo et Peretti-Watel, 2014 ; Braverman, 2018), la complexité des liens que le cancer entretient avec le genre reste encore mal saisie par l'analyse. À ne point douter, cette opposition apparente entre hommes et femmes dans la gestion de la trajectoire de la maladie fait sens. Mais diviser d'un point de vue sexué les 
parcours de santé se confrontent vite à l'éparpillement des identités et des technologies du genre (Lauretis, 2007). Si l'identité de genre (Alessandrin et E-Bellebeau, 2014) autorise à penser les masculinités et les féminités au prisme des phénomènes distincts et complémentaires plutôt stabilisés, comme l'assignation du sexe à la naissance, les technologies du genre mises en place par le sujet malade mettent en lumière une dynamique susceptible de faire bouger les lignes identitaires.

Directement inspirées de la pensée foucaldienne autour des «technologies du corps », les technologies du genre indiquent un ensemble des pratiques sociales et discursives qui produisent le sujet comme «genré ». Toutes ces voies que le genre emprunte pour s'exprimer - à savoir, le langage, les vêtements, la sexualité... -, régulent tant les représentations collectives que les techniques de production de soi. Dès lors, elles constituent un véritable instrument biopolitique qui produit la «différence des sexes » comme le socle des hiérarchies «naturelles » et, à la fois, un levier d'opposition qui appelle à la mise en place de stratégies. Dans la mesure où ces stratégies permettent de saisir des processus plutôt que des masculinités ou féminités statiques, elles favorisent l'analyse les territoires de formation des subjectivités de genre, tout en cartographiant un espace de résistance face aux stéréotypes sexués. Cette appréhension du genre par ce qui le construit et, de manière concomitante par ce qui le déséquilibre en permanence, inaugure une lecture des trajectoires de la maladie plus dynamique, à l'égard des masculinités et des féminités négociées, mises à l'épreuve par l'expérience cancéreuse (Meidani et Alessandrin, 2017 ; 2018). Tel que nous l'envisageons pour cet article, le genre ne se résume donc pas à des rapports de pouvoir, mais renvoie à des équilibres singuliers, à des «bricolages » identitaires que des maladies spécifiques mettent en jeu. Si ceci n'évince pas les inégalités qui traversent les rapports sociaux entre hommes et 
femmes, cette appréhension du genre aborde un aspect plus relationnel du concept (Théry, 2016).

Toutefois, l'analyse de l'expérience cancéreuse à travers ces deux concepts - identité et technologies du genre -, ne va pas de soi et pose la question de leur opérationnalisation. L'identité de genre permet d'appréhender la capacité du malade de se réaliser à travers telle ou telle activité - domestique, sexuelle, professionnelle, de loisirs etc. -, sans rompre totalement avec cet homme ou cette femme d'autrefois. Conçues comme support identitaire, ces activités sont normées et éminemment sexuées, mais aussi soumises au changement. Dans un rapport circulaire entre maintien de soi et mutation, les technologies du genre viennent simultanément parfaire et / ou défaire les habitudes sexuées, permettant d'éclairer ces dimensions de soi que les malades souhaitent préserver et celles qu'ils consentent à changer. En effet, la plupart du temps, les traitements engendrent une restriction des capacités physiques, des douleurs itératives, et / ou de la fatigue chronique (Eaker et al., 2011), obligeant le malade à repenser ses activités, son rapport au corps, à soi et à l'autre, selon la redéfinition de l'horizon du possible qui se dit dans ce que le malade est encore en mesure d'entreprendre ou est amené à réajuster, parfois même à abandonner. Quand ces ajustements incarnent encore une possibilité de prise sur la maladie, le genre adviennent comme une ressource. Ici la logique profane côtoie la crainte de la rechute (Bell, 2010) modifiant le rapport au temps pour ces survivants (Muzzin et al., 1994). Il n'est pas étonnant donc que le genre paramètre cette entreprise $d u$ «guérir», toutes relations et activités sociales étant conditionnées par ces corps sexués (Mock, 1998).

Toutefois notre recherche ne s'engage que pour partie dans une comparaison sexuée bicatégorielle. Pour partie car les subjectivités de genre se donnent à voir au pluriel, et devant compter sur d'autres facteurs de différenciation tels l'âge, la génération, l'origine, le statut 
social et / ou l'orientation sexuelle qui, dans une perspective intersectionnelle, entrent en résonnance avec le vécu de la maladie. Pour appréhender l'articulation entre expérience cancéreuse et genre, il paraît donc essentiel d'introduire la question des inégalités sociales.

Au terme de l'analyse, l'étude de cette articulation, cancer / genre, permet de dégager deux pistes interprétatives distinctes. Elles se déclinent différemment selon d'un côté, le travail du malade - c'est-à-dire ses activités quotidiennes et les logiques sous-jacentes qui supportent son identité et ce qu'elles impliquent en termes de changement; et de l'autre, les interactions soignant·e.s / soigné.e.s. Chacune de ces deux pistes est conditionnée par l'ordre du genre simultanément conçu comme injonctif, c'est-à-dire soumis à des principes de reproduction, mais également comme dynamique, autrement dit toujours susceptible d'étonner (Alessandrin et E-Bellebeau, 2014). Si l'adhérence à des modèles de genre peu ou prou stéréotypés peut être tenue pour pathogène, elle peut aussi apparaître comme un levier précieux d'adaptation face aux modifications induites par l'expérience cancéreuse. Quant aux relations de soins qui découlent en partie des protocoles de prise en charge, des caractéristiques cliniques, de différentes phases de la trajectoire de la maladie : pré-diagnostic, prise en charge, rémission, et des temporalités induites par les traitements -, elles sont dictées par des configurations de genre spécifiques qui se retranscrivent dans les consultations observées. Ces dernières oscillent entre reproduction normative et ajustements de genre situés.

\section{Objectif et méthodologie de recherche}

En termes d'objectif de recherche, il ne s'agit pas pour nous de constituer des typologies par pathologies cancéreuses, par degré de gravité ni même par catégorie sexuée. Notre objectif est d'examiner le regard que les malades en particulier, mais aussi les professionnel-le·s et les proches aidant·e·s portent sur les expériences de la maladie, et la façon dont ces expériences 
varient en fonction du genre. Au long de notre analyse, nous essayerons donc d'un côté, de mettre en perspective les logiques de subjectivation des malades à travers les normes de genre, et de l'autre, de rapprocher nos observations microsociales sur les interactions asymétriques soignant·e·s / soigné·e·s aux rapports de sociaux de sexe (Fassin, 2000). En ce sens, notre travail épouse une lecture ethno-épidémiologique du cancer, portant sur le travail du malade et les interactions de soins et, à la fois, une socio-anthropologie critique refusant toute forme de réductionnisme «culturaliste» qui attribuerait par défaut tel ou tel comportement de santé à telle ou telle « culture sexuée » (Massé, 1995).

En matière de méthodologie, précisons que les enquêté·e·s et les situations ont été choisi·e·s en fonction de leur capacité à favoriser l'émergence de la théorie, échantillonnage théorique, et ce processus, inspiré de la grounded theory (Glaser et Strauss, [1967] 2010), a été poursuivi jusqu'à la saturation des données. Plus concrètement, comme indiqué supra, pour cet article, seul le volet qualitatif des données françaises a été considéré.

Le propos s'appuie sur 400 séances d'observation in situ, menées sur une période cumulée de trois mois, au cours des consultations de soins, des séances de chimiothérapie, des visites dans les chambres de malades et des réunions de concertation pluridisciplinaire (RCP), dans 5 structures hospitalières situées dans 3 villes du sud de la France : Toulouse, Bordeaux, Aurillac. Ces établissements nous ont accompagnés vers des personnes majeures atteintes des 4 cancers les plus fréquemment enregistrés en France métropolitaine (INCa, 2017) : les cancers du sein, de la prostate, du poumon et du côlon / rectum. Constitué à partir de la file active de ces établissements, l'échantillon considère des patient·e·s pris·e·s en charge durant les 5 dernières années, et son élaboration permet de rendre compte de la variété des diagnostics et des traitements : chirurgie, radiothérapie, chimiothérapie, hormonothérapie, curiethérapie, ultrasons, surveillance active. 
Aux données observationnelles s'ajoutent 100 entretiens semi-directifs approfondis, d'une durée moyenne de 2 heures et équitablement répartis selon le genre, conduits pour l'essentiel auprès des patient.e.s considéré·e·s en amont dans le cadre des consultations. Vingt entretiens ont été réalisés auprès de leurs proches aidant·e·s et autant auprès des professionnel-le·s de santé impliqué·e·s dans leur prise en charge. Cancérologues, chirurgiens, radiothérapeutes, chimiothérapeutes, psycho-oncologues, infirmier-e.s et aides-soignant·e·s font partie de ce corpus. Le lieu de l'entretien était laissé à la discrétion des participant·e·s dans l'optique d'assurer des conditions d'échanges optimales. La majorité d'entretiens a été réalisée à domicile, et dans un quart des cas les partenaires, exclusivement des femmes, y étaient présentes.

Concernant les profils des patient.e.s, la plus jeune participante avait 37 ans au moment de l'entretien et le plus âgé 82 , alors que l'âge médian de l'annonce du diagnostic se situe aux alentours de 63 ans. Un peu moins des 3/4 des malades interviewés sont issus de classes moyennes / aisées avec un niveau d'instruction supérieur au bac. Quant à leur situation conjugale, l'échantillon couvre un panel fort varié, avec des participant·e·s majoritairement engagéee·s dans des relations hétérosexuelles. Aucun de ces malades n'est pris en charge en soins palliatifs et $5 \%$ sont en rémission complète. Ajoutons que notre enquête se déroule des semaines ou des années après l'annonce du diagnostic, auprès des « survivant·e·s » aussi bien qu'auprès des personnes chez qui la pathologie n'est pas installée de longue date, concernées par la phase aigüe du traitement. L'analyse produite exprime par conséquent cet état de faits, mettant en relief des trajectoires de maladie tantôt bien établies tantôt récentes et particulièrement mouvantes.

Fort de ce matériau, l'article revient sur les articulations multiples que le cancer entretient avec le genre, analysées en trois temps. Plus particulièrement, dans une première partie, nous interrogeons l'identité de genre et la reproduction des normes sexuées à laquelle recourt une 
partie des patient·e·s à partir de ce double paradoxe autour des femmes présumées «faibles » mais tenaces devant la maladie, et des hommes supposés «durs» mais affaiblis par leur cancer. Dans une seconde partie, nous mettons en perspective les ajustements situés que la maladie initie et distinguerons ce qui, en termes de masculinités et de féminités plurielles, contribue à la mise en place des stratégies négociées, plus ou moins efficaces, visant à tenir tête à la maladie. Enfin, dans une troisième partie, nous montrons comment les relations de soins oscillent entre reproduction normative et ajustements de genre situés.

\section{Femmes et hommes face au cancer : la reproduction des normes de genre}

Déjà observée par des recherches précédentes en la matière (Meidani, 2018), l'expérience cancéreuse est marquée par un double paradoxe. En contexte hétérosexuel, les femmes énoncent leurs résistances au cancer comme quelque chose de nécessaire, pour elles d'une part, mais également et surtout vis-à-vis de leurs proches (enfants, compagnons et parents âgés). Nous discernons ici ce « dynamisme excessif » décrit par Herzlich et Pierret (1984) qui se retranscrit dans l'investissement renouvelé dans le rôle de la mère, de l'épouse, de la professionnelle. Et même si cet investissement peut être perçu, par les patientes elles-mêmes, comme l'une des causes possibles de la maladie, dans le sens où l'individu en vient à négliger son corps surmené, le fait de pouvoir réinvestir ces mêmes rôles est conçu comme salutaire, la preuve par excellence d'une identité de malade dorénavant rangée dans le tiroir du passé.

À l'inverse, la majorité des hommes dissimule ou minimise ses problèmes de santé, alors même que ces patients apparaissent plus fragilisés que les femmes par l'épreuve du cancer. De nombreux témoignages recueillis illustrent ce déni de la maladie (Kleinman, 1988) qui se retranscrit dans l'absence de pratiques préventives, la non reconnaissance du caractère pathologique de symptômes, le temps écoulé entre les premiers signes et la consultation, les 
écarts en matière d'observance... De telles stratégies permettent à ces malades de ne pas remettre frontalement en cause le modèle de masculinité traditionnelle largement incorporé par la majorité d'entre eux dans le passé.

Si l'envie d'investir à nouveaux frais les rôles sexués antérieurs est identique entre les deux groupes des patient·es, les raisons de cet investissement et les activités visées ne sont pas les mêmes. Chez les hommes, ce sont les pratiques professionnelles, sexuelles et collectives (sportives, syndicales, de loisirs...) qui sont favorisées ; alors que chez les femmes un care revisité fait place à leur corps malade. L'investissement dans ses activités passées permet de se réaliser au-delà de l'identité de malade, souvent perçue comme stigmatisante, du moins chez les hommes (Amsellem et Gautier, 2006). Pour la plupart d'entre eux, le cancer est appréhendé avec la grammaire de la «gravité »(Galand et Salès-Wuillemin, 2009), alors qu'en matière de mortalité, la tumeur prostatique est située en troisième rang, loin des ravages causés par le cancer du sein, que les femmes préfèrent, malgré cela, percevoir comme une «affection curable ${ }^{7}$. Nous retrouvons ici les inquiétudes des hommes à se présenter comme des malades sans défense, l'appréhension des pannes érectiles, leurs difficultés à verbaliser leurs émotions, leurs craintes de se sentir inutiles ou en perte d'autonomie (Meidani et Bousigue, 2019). Il en résulte une minimisation des symptômes qui vient saper la capacité des hommes à transmettre leurs attentes et mettre en place des parcours de soins appropriés au plus près de leurs besoins.

Mais au-delà des disparités sexuées, l'analyse du matériau recueilli met en perspective deux observations. La première souligne l'ancrage sur le réel. Pour mesurer leur guérison, l'indicateur retenu par ces malades est leur capacité à maintenir leurs activités quotidiennes passées. Éminemment sexuées, ces activités sont censées contrer le sentiment d'une crise

\footnotetext{
${ }^{7}$ Selon l'INCa, en 2017, en France métropolitaine on enregistre 54062 nouveaux cas de cancer du sein et 48427 cancers de la prostate, alors que la même année, on répertorie 11913 décès dus au cancer du sein et 8625 au cancer de la prostate.
} 
identitaire (Herzlich et Pierret, 1984) initiée par la maladie. Si la guérison est foncièrement ancrée sur les possibilités du sujet à réintégrer sa vie d'autrefois, c'est parce que cette dernière constitue le signe par excellence d'une participation sociale restaurée, loin des avatars du cancer (Herzlich, 1984). La seconde observation souligne l'incorporation des attributs normatifs qui camoufle la construction sexuée des expériences de cancer, reproduisant de la sorte une logique de naturalisation. Cette dernière atteste du fait que dans des sociétés profondément structurées par le genre, la dynamique socialisatrice et intégratrice des rapports sociaux de sexe qui participe vigoureusement au travail du malade, avance masquée.

Un double élément mérite d'être souligné à ce niveau. Du côté des hommes, nier le caractère pathologique des symptômes et continuer à s'investir dans leurs activités passées devient un moyen de nier la maladie même, ce qui les propulse dans le rôle d'un malade «passif », placé en retrait du parcours de soins. En revanche, du côté des femmes, composer avec l'identité de malade et la fragilité qui en découle, apprendre à «cohabiter » avec la maladie devient le socle d'une attitude d'auto-surveillance active, voire combative, autrement compatible avec la perspective d'une rémission totale. Nous retrouvons ces tendances dans d'autres analyses comme, par exemple, celles relatives au cancer du sein chez les femmes qui ne souhaitent pas s'enfermer dans des identités de malade (Jacques, 2009 ; Tarantini, Gallardo et Peretti-Watel, 2014), ou encore chez les hommes atteints du cancer de la prostate, où le sentiment d'une masculinité faillible semble esquisser des ruptures dans les scripts de la masculinité traditionnelle (Braverman, 2018).

Nathalie $^{8}$ a 55 ans. Elle est coiffeuse. Atteinte d'un cancer du sein, elle a subi une mastectomie curative ${ }^{9}, 2$ ans avant notre entretien. Malgré ce corps mutilé, Nathalie n'a pas voulu «se faire refaire le sein ». Durant les séances de chimiothérapie qui ont suivi sa

\footnotetext{
${ }^{8}$ Conformément aux règles de confidentialité et d'anonymisation, tous les prénoms et noms mobilisés dans le cadre de cet article correspondent à des pseudonymes.

$930 \%$ des femmes interviewées se trouvent dans le même cas. Le pourcentage correspond aux données nationales disponibles (Observatoire sociétal des cancers, 2014).
} 
mastectomie, elle se souvient de ses parents qui répétaient sans cesse aux enfants « ne fatiguez pas maman, elle est fragile ». À l'inverse, leur père, Claude, artisan bijoutier à la retraite, 63 ans, lui-même atteint d'un cancer de la prostate était plus souvent appelé en renfort, y compris dans des exercices davantage physiques comme «le jardinage ou le bricolage. » Ces dernières sont décrites par le malade comme sa «soupape de sécurité ». C'est de la sorte que Claude tient à distance le sentiment d'inutilité qui le guette. Mais, à vrai dire, Claude n'aime pas parler de sa maladie, et encore moins de sa prostatectomie, et durant le court entretien qu'il nous accorde, il ne prononce jamais le mot cancer. D'ailleurs, il reste très évasif quant au traitement qu'il suit : une hormonothérapie que, selon son épouse, «il vit très mal », à cause d'une gynécomastie iatrogène «qui est venue s'ajouter aux fuites urinaires. Depuis, Claude s'est refermé sur lui-même ». D'après Nathalie, compte tenu des effets indésirables, son mari « souhaite arrêter le traitement mais le médecin n'est pas d'accord. » Coupant court à nos relances, Claude se contente de commenter en deux mots la tension avec le cancérologue : «ce n'est rien, je vais chez le médecin il me dit ce qu'il faut prendre, puis voilà ».

Le travail du malade n'est pas lisse et les ambiguités qui l'animent sont nombreuses. Les ajustements d'activités qui en résultent sont alors généralement perçus comme contraints par les malades. Cette manière d'être un homme ou une femme avant tout « malade » est souvent jugée comme insatisfaisante, incomplète, moindre ou inférieure par rapport aux canons de la masculinité et de la féminité traditionnelles qui font, toujours et malgré tout, office de référence. Faisant l'hypothèse qu'un homme qui a de la poitrine, souffre d'incontinence ou n'a plus d'érection, n'est plus un homme, au même titre qu'une femme sans sein n'est plus une femme, prostatectomie et mammectomie vont jusqu'à initier le deuil des identités de genre traditionnelles. De nombreux hommes vivent les suites de la maladie et les limites qu'ils explorent au quotidien comme des situations de mise en échec de leur virilité. De tels échecs ouvrent vers une masculinité de moindre valeur orientant le parcours de soins. Didier, 
63 ans, enseignant-retraité, divorcé, qui a refusé la prostatectomie, nous raconte à ce propos : «j'avais entendu dire qu'après l'opération on se sent plus un homme. J'ai donc préféré les radios, et même comme ça, c'est difficile!»

Face à ces constats, nous sommes en mesure d'avancer certains éléments interprétatifs. Le premier se déploie autour de l'idée que les normes de genre, dans leur reproduction, adviennent comme des éléments sécurisants, supports de résistance dans une épreuve qui impose au sujet malade un corps faillible et changeant. À cet égard, le recours à des normes de genre connues peut représenter une ressource importante pour soi. Marie, 63 ans, femme au foyer, mère de deux enfants qui ne vivent plus avec elle, elle a eu recours à une opération conservatrice du sein et des séances de radiothérapie. Marie voit dans son mari quelqu'un « de fort qui l'a toujours soutenue [...] Heureusement qu'il est là», dit-elle. Mais en avançant, Marie nuance son témoignage :

«Xavier [son mari] est incapable de rentrer dans un hôpital sans tomber dans les pommes, et ça a toujours été. Quand il m'amenait à l'hôpital il restait dans la voiture, et sur la route du retour j'évitais de lui parler de ça [maladie]. Maintenant c'est le taxi qui m'amène [...] Je prie Dieu pour que rien ne lui arrive...»

Le deuxième mécanisme de reproduction normative que le genre convoque dès lors que la maladie cancéreuse perce la trajectoire d'une vie, c'est autrui. Un théâtre en particulier déploie de manière sensible cette répartition traditionnelle des normes de genre, celui de la sexualité (Bajos et Bozon, 2008). Jean, 63 ans, informaticien toujours en activité au moment de l'entretien, évoque avec embarras les difficultés qu'il a non seulement à avoir une érection mais également à la maintenir suite à sa curiethérapie : «Et ce n'est pas à cause de mon âge... » L'enquêteur entrevoyant la difficulté de Jean à mettre des mots sur son expérience, le relance : «Avez-vous recours à une aide médicamenteuse ?» Jean encaisse plus qu'il ne le 
confirme avec un signe de la tête. Lors de l'entretien qui suivra cet échange avec sa seconde compagne, d'une vingtaine d'années plus jeune que lui avec laquelle il n'a pas d'enfant, la question de la sexualité s'avère être «importante ». Ici, le traitement médicamenteux relève plutôt «des devoirs de conjugalité », commenté en ces termes par le cancérologue qui suit Jean. Dans l'interview réalisée avec ce dernier, nous comprendrons que Jean subit des injections intra-caverneuses 3 fois par mois, pour pouvoir avoir une érection. Après avoir essayé «le viagra et la pompe à vide, la pose d'une prothèse pénienne est envisagée ». La tentative de continuité dans les normes de genre (Esteve-Bellebeau, 2018) rassurerait donc non seulement les soignés mais également les proches qui, dans une logique essentialisante, encouragent les malades à continuer à inscrire leurs activités quotidiennes dans des registres de genre bien distincts.

Enfin, un troisième point apparaît régulièrement dans les observations, notamment autour des ressources mobilisables pour faire face au cancer. Si les femmes mettent très souvent en avant des pratiques de santé complémentaires et alternatives, comme autant d'éléments d'accompagnement de santé réconfortants (Cohen et al., 2015); les hommes, quant à eux, ont plus spontanément recours aux indices médicaux et aux avancées technologiques qu'ils conçoivent comme des éléments probants. Prendre en considération l'effet performatif des tests du PSA (Prostate Specific Antigen), des biopsies, des IRM (Imagerie par Résonance Magnétique), des scintigraphies et d'autres examens revient à souligner que les registres des soins oncologiques restent excessivement clivés du point de vue du genre, tant du côté des soigné·e·s que des soignant.e.s. Pour le dire avec les théoriciennes du care (Molinier, 2013 ; Brugère, 2014), le travail de soins connaît des fortes résonnances de genre que les épisodes cancéreux mettent également à jour.

Extrait de carnet ethnographique en date du 17 mai 2016 à Bordeaux. 
Annie, qui accompagne son mari Gérard chez le radiothérapeute, explique avec précision au susmentionné qu'en raison des complications digestives et urinaires du traitement, elle s'emploie dorénavant à confectionner des recettes particulières dont elle a entendu les bienfaits sur la santé. Tout à l'opposé, son mari s'empresse de tendre au praticien une prescription, coupant sec la conversation: «plus sérieusement docteur, avez-vous reçu les résultats de mes derniers examens ?» Tout comme Gérard, la majorité absolue des hommes reçus par le praticien $(\mathrm{N}=8)$ ce jour était accompagnés de leurs épouses ou de leurs enfants - par opposition à la plupart des femmes venues seules -, et les interventions des accompagnants dans les échanges avec le soignant étaient bienvenues, du moins aux yeux de ce dernier.

Si la socialisation de genre participe de la diffusion des stéréotypes sexués, l'analyse nuance leur généralité présumée. On note alors qu'à l'entreprise de soins s'adjoint une seconde entreprise que l'on pourrait qualifier d'inversion de genre, particulièrement opérante en régime hétérosexuel : la «virilisation» des femmes malades et la « féminisation» des hommes atteints de cancer. D'un côté, les femmes endossent des responsabilités relationnelles ou familiales, propre au travail du care. Elles expriment régulièrement qu'elles doivent «tenir » pour «faire tenir » leurs proches. Par-là même, elles se retrouvent en situation de résister au cancer. D'un autre côté, le corps masculin, réceptacle des soins, se vit comme diminué. Cette dissymétrie de genre Certes, ayant à vivre l'expérience de la domination dès leur plus jeune âge, s'en émancipant partiellement dans la période récente, les femmes conscientisent plus aisément que les hommes l'identité du malade. Ce qui revient à dire que cette « femme-objet » est davantage prédisposée à adopter le rôle du « sujet malade \#, à l'opposé d'un « homme-sujet » plus enclin à réduire ses pratiques de soins à leur dimension curative la plus élémentaire, endossant le rôle d'un " objet malade » (Meidani, 2005). Faisant le constat de ce double paradoxe, l'analyse-montre que la 
reproduction de la bipartition traditionnelle des rôles de genre rassure non seulement les soignés mais également les proches et les soignants qui, dans une logique essentialisante, encouragent les malades à continuer à inscrire leurs activités dans des registres de genre bien distincts.

\section{Expériences cancéreuses et arrangements de genre. Parcours de soins, parcours de} sens

Si l'analyse précédente demeure éclairante de nombreuses situations rencontrées, elle ne permet pas de rendre compte de la complexité des liens que le cancer entretient avec le genre. Certes dans une dynamique de reproduction des normes sexuées traditionnelles, la manière de «faire genre » advient comme l'une des conditions de réalisation de soi qui permet de tenir à distance l'identité du malade : une façon d'être «pleinement» cet homme ou cette femme d'autrefois. Mais pour certains patient·e.s, l'expérience de genre est reconnue comme un facteur déclencheur du cancer qu'il convient de «corriger», afin de rompre définitivement avec cet homme ou cette femme malade. Ici le travail de reconversion de soi initié par l'annonce de la maladie, amène à des réadaptations identitaires attachées à la dissolution, plus ou moins partielle, d'une identité de genre jusque-là perçue comme stabilisée, mais d'ores et déjà reconnue comme préjudiciable.

Dans ce cadre, les technologies de genre mobilisées dans la gestion de la maladie incarnent la clé de voute du processus de guérison. Les stratégies adoptées sont alors exprimées dans la diminution du nombre d'heures réservées aux tâches domestiques, le changement du partenaire et parfois du statut marital, l'allégement des charges du travail ou la reconversion professionnelle, le changement d'équipe médicale, le deuil d'un projet d'enfant et, même parfois, la réorientation sexuelle. 
Deux pistes interprétatives dialectiques peuvent à ce stade de la discussion être repérées. La première concerne les féminités et les masculinités contre- ou non-hégémoniques, c'est à dire minoritaires, qui parviennent à puiser dans leurs expériences sociales passées les ressources nécessaires afin de négocier les attentes de genre face à un corps malade. Nous retrouvons ici les portraits des femmes féministes et des figures d'hommes progressistes. Dans ces cas-là, c'est l'antécédence de l'identité de genre qui permet d'intervenir sur la gestion de la trajectoire de la maladie. Les patient·e·s les plus jeunes et diplômé·e·s, généralement issu·e·s des classes les plus aisées de notre échantillon, sont les plus représenté·e·s dans cette catégorie.

Pierre, 67 ans, médecin généraliste, parent de cinq enfants, qui vit avec sa deuxième épouse, a été «opéré par rabot» quelques mois avant l'entretien. «On se laisse aller à un autre type de sexualité ce qui est très plaisant pour ma femme, même si nous n'avons pas attendu le cancer pour savoir qu'on pouvait avoir du plaisir autrement [qu'avec une sexualité pénétrative]. » Plus que l'âge de Pierre c'est son niveau d'études, sa génération de baby-boomers et, par là même, l'antécédence de ses expériences plus égalitaires avec ses anciennes partenaires, qu'il convient de retenir ici comme éléments éclairants.

Du côté de la population féminine un profil comportemental équivalent apparaît. Alice a 54 ans. Ingénieure de recherche, elle vit avec sa compagne et son fils depuis 15 ans. Atteinte d'un cancer du sein déclaré quelques semaines avant notre rencontre, Alice compose avec l'idée d'une ablation totale ou partielle et écarte l'idée d'une reconstruction mammaire. «Ça fait 25 ans que je milite dans des associations féministes. Ce n'est pas moi qui vais venir dire que c'est là [elle montre sa poitrine du doigt] que sont ma force et ma féminité [...] Je n'ai rien à faire qu'ils [les médecins] me traitent de butch, de trans, ou qu'ils pensent que j'ai été abusée sexuellement [...] Si en France on était moins réfractaire à la mammectomie préventive, je le ferais... pour retrouver mon corps d'enfance. » 
Dans cette première piste interprétative esquissée à travers les témoignages présentés plus haut, les ressources culturelles, militantes et plus généralement expérientielles qui autorisent des pas de côté vis-à-vis des normes traditionnelles de genre, ouvrent avec plus de souplesse au processus de réaménagement des activités quotidiennes face aux changements imposés par le cancer. Mais d'autres arrangements de genre sont plus directement dus à l'annonce du diagnostic et au protocole de prise en charge, qui deviennent alors des facteurs déclenchant des stratégies mises en œuvre. Nous passons ainsi à la seconde piste interprétative qui prend le cancer comme référence antécédente des arrangements de genre. Il s'agit là des patient·e·s qui, du fait de leur cancer, renégocient et adaptent leurs activités et leurs relations avec leurs proches. Ici l'expérience cancéreuse prend place et sens en accord avec ce genre auquel le malade aspire et dont il s'inspire. La maladie advient alors comme une ressource, grâce aux contraintes qu'elle instaure, et fait place à des configurations de genre, des relations et des activités revisitées qui confèrent une nouvelle forme de cohérence identitaire. Ainsi le genre apparaît comme un élément fondateur à partir duquel s'organisent la production de ruptures, la genèse de nouvelles intelligibilités et l'inscription de la maladie dans une continuité biographique (Christias, 2009).

Anna, 50 ans, enseignante d'espagnol au lycée, divorcée et mère d'un enfant, cherche à composer avec un cancer du sein annoncé quelques semaines avant notre rencontre. «Il faudra bien faire avec », nous dit-elle. Pour Anna, sa féminité va forcément être «métamorphosée». Et même si elle laisse ouverte la perspective de la reconstruction mammaire, elle appréhende la prise de risque que ça représente. Anna consulte régulièrement des sites et des blogs ${ }^{10}$ des personnes atteintes du même cancer qu'elle, afin de «bien faire quelque chose» de ce qui lui arrive. Elle se dit aussi «prête à mettre de l'ordre dans le quotidien [...] Vous savez lorsque le corps reprend ses droits il y a beaucoup de choses qui

\footnotetext{
${ }^{10}$ Même si un nombre non négligeable de patients dit avoir recours à internet pour relever des informations en lien avec sa maladie, ce comportement a été plus fréquemment relevé chez les femmes.
} 
passent en second plan [...] Et si mes collègues, mon partenaire ou mes amis ne le comprennent pas, et bien il me faudra changer de collègues, de partenaire et d'amis. » Décrivant les changements que le cancer initie dans sa vie, Anna envisage de s'accorder «des soins de messages », réaliser un «tatouage réparateur pour cacher sa mammectomie », faire ses courses sur internet et même se mettre à temps partiel. Mais à l'opposé de la majorité des femmes interviewées, elle se montre plus critique face aux associations : «Non... ça ne m'intéresse pas, j'ai essayé mais je n'ai rien à faire d'aller prendre le thé avec des vieilles dames. Avoir eu un cancer ne suffit pas pour... disons qu'il faut un peu de pédagogie. J'ai ma fille. On est très fusionnelle. »

Se faire aider par son enfant, faire dorénavant ses courses sur internet, recevoir le soutien d'autres personnes qui traversent cette même épreuve dans le cadre des structures associatives ou sur le net, découvrir des soins complémentaires et alternatifs, se mettre à temps partiel... toutes ces initiatives permettent d'intégrer la maladie dans son histoire de vie (Bataille, 2003). Les patients ne restent pas donc passifs devant l'épreuve du cancer. Ils cherchent à relativiser ces expériences et, pour ce faire, ils ont recours à diverses stratégies. Nous pouvons les regrouper dans trois grands ensembles qui font écho aux technologies du genre, et prennent appui sur des logiques distinctes. Selon la première, largement encouragée par le corps médical et les proches, la mise à l'épreuve de sa propre masculinité / féminité est considérée comme «un prix à payer» pour être encore en vie. C'est ce que nous raconte Candice, 63 ans, secrétaire à la retraite, mariée et mère d'un enfant, en suivi chimio-thérapeutique : «Je n'ai pas le choix. C'est le prix à payer. Et ma foi, par rapport à d'autres femmes atteintes de la maladie, je n'ai pas à me plaindre. J'ai une collègue qui a eu le même cancer et qui a refusé la mastectomie. Elle en est morte. »

Selon la deuxième logique, de nombreuses personnes malades, notamment les plus âgées de notre échantillon, se réfèrent au processus de déprise (Meidani et Cavalli, 2018 ; 2019 ; 
Meidani et Bousigue, 2019) déjà engagé pour elles, bien avant la découverte du cancer. Devant la réalité amère du vieillissement et la prise de conscience de ce corps qui s'épuise peu à peu, les patient·e·s accueillent les répercussions de la maladie et du traitement avec un certain stoïcisme. C'est devant la proximité de la mort que le cancer est alors relativisé. C'est ce que l'on retrouve par rapport au deuil d'une certaine sexualité, «celle d'avant », ou encore d'une carrière professionnelle que les hommes des classes aisées relatent avec fierté.

La troisième logique fait place aux cancers à «bons pronostics » - à l'image de la tumeur prostatique -, aux épisodes cancéreux «digérés » intervenus plusieurs années avant l'entretien, ou encore à la présentation du protocole de soins toute suite après l'annonce du diagnostic - ce qui participe à relativiser la «mauvaise nouvelle » (Ménoret, 1999 ; Bataille, 2003). Camille, 44 ans, relate : «le médecin a été très bien. Il m'a dit 'voilà, nous avons une zone affectée. Et voilà ce qu'on peut faire pour la traiter'. Et ça, ça rassure. »Cette triple logique qui anime les stratégies des malades nous permet de discuter la notion de rupture biographique. Si sa valeur herméneutique n'est plus à démontrer, relier automatiquement l'annonce du cancer à une rupture biographique ne permet pas de rendre compte de la complexité de l'entrée dans la maladie (Voegtli, 2004) et de restituer la pluralité des vécus.

Les reconfigurations de genre qui en découlent n'esquissent pas un processus individuel : l'entourage participe pleinement à ce remodelage du quotidien en soutenant le malade ou, au contraire, en lui faisant savoir sa réprobation. En effet, les attentes et les points de vue des proches pèsent sur le travail du malade. Ainsi la mère de Nadine qui a pris soin de sa petite fille lors de l'hospitalisation de sa mère, se montre très critique face à la consommation tabagique de sa fille. Mais Nadine ne l'entend pas de cette oreille : «c'est la clope qui me permet de tenir, parce qu'entre mon ex et les collègues du boulot...» 
C'est parce que l'expérience du cancer peut conduire l'individu à remettre en cause bien des choses qui allaient de soi jusqu'à son avènement, en particulier le primat accordé à une certaine carrière de genre (Meidani et Alessandrin, 2018), qu'elle est également susceptible de remodeler les aspirations des « survivants ». Antoine, 52 ans, est chercheur. Lors de notre rencontre, nous parlera des bifurcations induites par sa maladie, touchant à sa vie affective et sexuelle ainsi qu'à son projet parental. Faisant définitivement le deuil de ce dernier, Antoine s'affiche dorénavant comme «gay», et décrit les difficultés pour son entourage familial d'accepter ses choix.

Tandis que nous soutenons qu'une masculinité dominante peut être considérée comme un «facteur de risque » pour contrer efficacement la maladie, nous notons également que la définition de genre n'est ni homogène ni inflexible. Des configurations «souples » de la masculinité, majoritairement enregistrées chez les plus jeunes de nos participants, donnent suite à des réajustements du genre significatifs, observés aussi au sein de la patientèle féminine. Parfois ces réajustements précèdent la maladie. D'autrefois ils donnent lieu à des reconfigurations qui prennent place à mesure que les normes et les valeurs sur lesquelles s'appuient la masculinité ou la féminité conventionnelles s'effilochent, à cause de l'avènement du cancer. L'analyse de ces réajustements permet de montrer dans quelle mesure et sous quelles conditions, les malades - accompagnés par leurs proches et les professionnels, parfois même malgré ou contre eux - peuvent s'engager dans des formes de résistance aux normes de genre dominantes, en dépit des situations de vulnérabilité dans lesquelles ils sont projetés.

Plus spécifiquement, la reconnaissance du sein et de la prostate comme des éléments identificateurs de l'orientation sexuelle et du genre conditionne l'expérience de tou·te·s les malades qui essaient de construire leur rapport avec les corps post-chirurgicaux au-delà des modèles traditionnels de genre. Dans la mesure où les changements physiques que le 
traitement initie, troublent la correspondance entre traits anatomiques et appartenance de genre (Greco, 2016), ils mettent à mal les représentations dichotomiques des corps masculins

et féminins binaires. À ce titre, les traitements des cancers sexués peuvent être interprétés comme des altérations de la binarité.

Si par leur localisation, ces cancers - plus que d'autres tumeurs -, marquent la différence entre le masculin et le féminin, les patient·ess ne se plient pas toujours à la division binaire des corps sexués. Parfois, le refus des malades de recourir à une reconstruction mammaire ou à des dispositifs de restauration érectile représente une volonté de dépasser les modèles sexués, en se positionnant en dehors de la binarité. Ces corps «différents » sont donc perçus comme un expédient pour se soustraire aux injonctions du genre. Ils peuvent même exprimer la volonté de revenir en arrière, vers un «corps d'enfance » qui ne véhicule pas de marqueurs distinctifs de la masculinité ou de la féminité. D'autres fois, ils évoluent sous le signe d'un âge avancé qui garde à distance le jeu de séduction, de sexualité ou encore l'envie de s'approcher d'une apparence «masculine » ou « féminine ». En outre, dans de nombreux cas, le refus de recourir à certaines techniques médicales incarne un moyen de réduire des prises de risque supplémentaires, et une façon de décider encore du sort de sa vie. La polysémie qui en résulte trace les contours de nouveaux parcours de sens à travers lesquels les soignant·e·s gagneraient à parcourir l'histoire de ces corps cancéreux.

\section{Genre, cancer et expériences situées : les interactions soignant·e·s / soigné·e·s}

Insistant sur l'expérience subjective du cancer, la littérature a mis de côté les interactions soignant·e.s / soigné.e.s et l'analyse des rapports sociaux de sexe qui s'y déploient. Or, le genre concourt à définir l'expérience de soins (Ahmed et Bates, 2007). Plusieurs modalités d'interactions peuvent être décrites, sans toutefois qu'elles apparaissent immuables car, 
généralement et pour la plupart, les interactions de soins s'inscrivent dans le temps et ne sont jamais complétement les mêmes d'un rendez-vous à l'autre. Dans les observations in situ et les entretiens qui les ont suivies, il apparaît clairement que plus les technologies et l'identité du genre des soignéee-s sont conformes aux attentes et aux expressions du genre des soignant·e·s, plus les relations de soins sont apaisées et sécurisantes. À l'inverse, plus les technologies et l'identité du genre des soigné.e-s s'éloignent des attentes et des expressions du genre des soignant·e.s, plus les interactions de soins sont compliquées parasitant de la sorte le parcours de prise en charge.

Myriam a 39 ans et vit seule avec sa fille. Elle détient un master en gestion et dirige le pôle de ressources humaines d'une entreprise privée. Porteuse d'une mutation génétique BRCA 1, Myriam est atteinte d'un cancer «compliqué ». À la suite de sa mastectomie, elle vient voir pour la première fois le docteur Dupont. Elle en avait entendu parler «par réputation », une réputation que la patiente ne manquera pas de mettre en perspective avec «l'homosexualité » du praticien. Dans le cadre de la séance d'observation, Myriam semble rassurée par l'attitude du cancérologue qui, très vite, quitte sa chaise derrière le bureau et vient s'asseoir à ses côtés prenant un papier et un stylo. En écoutant les réponses de Myriam qui lui permettent de situer son profil, bien plus que les caractéristiques cliniques de sa tumeur, il commence à dessiner les différentes séquences du protocole de sa prise en charge, avant de le soumettre à la patiente pour «validation ».

Dans l'entretien qui suivra la consultation, Myriam nous précisera que c'est bien la masculinité incarnée par son ancien praticien qui l'a poussée à chercher un autre oncologue qui, pour reprendre ses termes, «ne se la jouait pas gros mâle viril ». Pour lui, tout comme pour le chirurgien qui a opéré Myriam, la perspective d'une reconstruction mammaire représentait un «vrai plus » qui allait lui permettre, non seulement, de rétablir la symétrie 
mais aussi d'accéder à une poitrine "plus conforme aux standards de beauté ». Mais aux yeux de Myriam, ce modèle de virilité symbolisait une source de tension rendant compliqué le suivi médical, d'autant qu'il entrait en écho avec l'establishment médical qui invite les femmes à «rester féminines, se maquiller et cacher leurs corps asymétriques à travers des prothèses et des lingeries adaptées ». Ces injonctions exaspéraient Myriam qui, depuis sa maladie, laisse ouverte la question de sa réorientation sexuelle, constatant que c'est son amie lesbienne qui l'a « le plus soutenue durant cette épreuve ».

Le récit de Myriam esquisse en filigrane la suspicion avec laquelle les soignant·e·s accueillent le refus d'une reconstruction mammaire, notamment lorsqu'il émane des plus jeunes patientes, au moment même où près de $75 \%$ des femmes qui ont effectué une mastectomie refusent de «passer sous le bistouri» (Henry, Baas et Mathelin, 2010). Tenant tête à l'autorité médicale, Myriam présente son choix comme l'indice «d'une posture active » de malade dont elle se réclame. Sa décision lui permet de mieux gérer certains temps de la maladie - à l'image des séances chimio-thérapeutiques - tout en poursuivant ses activités professionnelles, et s'inscrit dans le refus de se mettre en congé maladie le long de son traitement.

Quant au cancérologue Dupont, son homosexualité n'est pas forcément compatible avec les attentes de toutes les patientes : «certaines d'entre elles ont préféré ne plus être suivies par moi à cause de mon homosexualité », nous confie-t-il. Lors de l'entretien, le cancérologue fait référence à une technique hormono-thérapeutique de 15 ans qui enregistre actuellement un taux d'observance très faible. Mais la patientèle suivie par l'oncologue fait figure d'exception, un élément connu par l'intervieweur qui l'interpelle sur ce point :

«On peut faire faire beaucoup de choses aux malades à partir du moment où on les accompagne », lui répond-il [...] Je pense n'avoir jamais dit à une patiente que le 
cancer est une maladie incurable, je préfère leur dire que "je ne sais pas les guérir", ce qui permet de se placer sur un pied d'égalité. C'est essentiel pour favoriser l'alliance thérapeutique. »

La normativité des codes de genre ne conditionne pas donc de manière homogène et automatique les interactions de soins. En entretien informel dans la salle d'attente, Patricia et son mari, que nous suivons alors en consultation, nous décrivent l'oncologue en ces termes : «C'est un bon! On ne cherche pas un psychologue quand on vient ici mais un médecin compétent, c'est ça qui compte. »

Patricia, 49 ans, femme au foyer, est suivie pour un cancer du sein, et a déjà eu recours à une reconstruction mammaire. En consultation, elle accompagne son mari, âgé d'une bonne dizaine d'années de plus, atteint d'un cancer de la prostate et également pris en charge dans le même établissement de soins. De classe sociale aisée, ils vivent avec leurs trois enfants. L'oncologue Canus, 39 ans, qui suit le mari de Patricia, nous décrit leur profil en ces termes : «Vous verrez, il s'agit d'un couple dont le mari est PDG d'une grande boîte. Avec lui, il faut montrer qu'on en a, sinon il ne nous considère pas comme un bon médecin. Il est souvent en demande, du coup il faut ajuster le protocole, et ça prend plus de temps. » Lors de la consultation, c'est le mari qui prend la parole pour lui et le plus souvent pour sa femme. Pour évoquer sa sexualité, il s'adresse en ces mots au médecin : «Entre hommes docteur, on se comprend... »

En cancérologie, la lecture biomédicale de la sexualité, souvent phallocentrique (Tiefer, 1994) et teintée d'hétéronormativité pénétrative, se restreint à la seule capacité d'érection. En assimilant la sexualité à l'hétérosexualité, la prise en charge des questions sexuelles participe à l'invisibilisation des minorités sexuelles, et renforcent l'asymétrie de genre entre les partenaires hétérosexuels. Là aussi les technologies du genre mobilisées par les praticien.ne·s 
diffèrent selon l'identité sexuée du malade. Ainsi pour les hommes, les soignant·e·s - tous sexes confondus - mettent souvent en avant les activités professionnelles et la sexualité, même si le tabou demeure pour les plus âgés d'entre eux (Arrington, 2004). En revanche, ces deux sphères d'activités passent en second plan - quand elles ne relèvent pas de l'impensé lorsqu'il s'agit de prendre en charge une femme, pour laquelle ce sont surtout des questions de santé reproductive qui seront considérées, dès lors que son âge le permet et qu'elle n'est pas déjà parente.

La prise en compte du point de vue de l'oncologue permet de compléter l'analyse :

«Même s'il y a un effet générationnel côté soignants, c'est surtout une question d'expérience [...] Avec 15 ans de psychothérapie derrière moi je considère pouvoir accompagner les malades, mais ce n'est pas le cas de tout le monde. Une étude récente montrait qu’un soignant sur quatre est médiqué : anxiolytiques, somnifères, antidépresseurs etc. Tu ne peux pas soigner quand tu es malade. Là encore dans cet établissement nous avons le luxe de pouvoir prendre le temps avec les patients, mais ce n'est pas le cas à l'hôpital [...] Tu veux que je te dise pourquoi les femmes ne se plaignent pas, parce que personne ne s'y intéresse, parce que tout le monde pense qu'elles résistent à la douleur. Donc leurs plaintes, elles les avalent et elles tracent, et ça les rend plus fortes. »

Dans les mots de ce cancérologue, comme dans les récits de ses collègues, la doctrine du moral qui prend les contours d'une idéologie susceptible d'augmenter les chances de survie, se présente comme structurellement attachée au féminin, et à ce qu'elle implique comme voie de guérison possible (Saillant, 1988). Mais à la lecture de ces témoignages, nous concluons

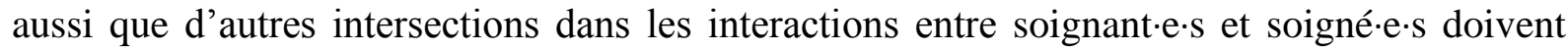
être prises en compte dans l'analyse genrée des parcours de soins : la sexualité - réelle ou 
supposée - des acteurs réunis, la classe sociale, la génération, les parcours de santé des soigné·e·s, tout comme ceux des soignant·e.s, ainsi que les conditions matérielles d'exercice de leurs fonctions.

À l'opposé du cancer du sein, la prise en charge de la tumeur prostatique représente parfois l'occasion d'endosser des attitudes de genre performatives. «Faites ce que vous voulez docteur mais je ne veux pas de postiches. Je veux avoir la gaule », précise Karim, un ouvrier retraité de 62 ans, «d'origine tunisienne ». L'oncologue ne proposera pas à Karim de consultations chez le sexologue du service, et lors de l'entretien de débriefing qui suivra la courte consultation, il insistera sur les consommations alcoolo-tabagiques du malade. À l'égard de ces malades, les praticiens adoptent volontiers une posture virile qui se caractérise par une faible empathie, alors que les infirmières n'investissent pas toujours le travail relationnel. C'est alors avec les hommes de classe populaire, qui plus est adeptes de pratiques alcoolo-tabagiques, que les consultations sont les plus standardisées et les plus courtes. En revanche, face à des majoritaires - employés et professions intermédiaires -, et notamment les malades issus des classes aisées, le contenu de consultations varie en fonction des éléments biographiques apportés par les patient·e.s, permettant ainsi aux soignant·e·s de moduler les modalités de prise en charge. Mais au-delà d'un travail de soins qui se heurte à la dévalorisation sociale du care (Tronto, 1993), et de la place des conduites dites à risques dans les protocoles de prise en charge, le dernier extrait ethnographique laisse aussi entrevoir les inégalités qui structurent l'offre de soins, avec des traitements sur la sexualité, par exemple, plus ou moins accessibles selon les zones du territoire.

Un constat analogue concerne le dispositif d'annonce dont les malades du cancer bénéficient, en principe, depuis 2006. Si les femmes interviewées se sont vues, dans leur majorité, proposer des échanges avec une infirmière et des soins de support avec une psychologue ou une assistante sociale, rares sont les hommes qui ont bénéficié de tels dispositifs - notamment 
dans les zones rurales. Cette observation est d'autant plus importante que le regard que les patient.e.s portent sur les interactions semble dépendre moins de la localisation du cancer ${ }^{11}$ que d'un prognostic initial dit « mauvais » et des effets indésirables des traitements reçus : état de fatigue cliniquement significatif, douleurs, altération de l'image du corps (VICAN, $2018^{12}$ ). En accord avec ce dernier point, l'analyse montre que c'est l'annonce du diagnostic qui cristallise le plus de tension. Elle est jugée «brutale» chez les femmes les moins diplômées et disposant généralement des faibles revenus - et notamment les plus jeunes d'entre elles, âgées de moins de 50 ans.

$\mathrm{Au}$ terme de cette troisième partie, il est possible de conclure qu'au sein des expériences de soins, les féminités et les masculinités plurielles, induites par des rôles sociaux variés, doivent être comprises plutôt comme des configurations pratiques qui prennent sens et se déploient de manière relationnelle. Loin d'être le fruit du hasard, les positions des acteurs impliqués dans le soin sont intriquées avec des questions de genre, de santé, d’âge, de génération, de sexualité et de classe. Si la prise en compte des effets liés à ces déterminants révèle des hiérarchies entre les masculinités et les féminités contribuant à classer les malades entre eux, c'est avant tout la force socialisatrice du genre qui fait la lumière sur l'expérience cancéreuse de soins. Pour le dire autrement, parmi tous les determinants qui conditionnent l'expérience de la maladie, c'est le genre qui enregistre les effets les plus clivants.

Vécus à la fois comme l'une des causes possibles du cancer et comme l'une des conditions de sa guérison, les rapports sociaux de sexe qui se déploient au sein des interactions de soins façonnent les liens que malades et professionnel-le·s entretiennent avec la maladie. Entre potentiel thérapeutique et potentiel pathogène du genre, logiques profanes et logiques médicales du soin, les normes sexuées saisies dans un processus tantôt reproductif tantôt

\footnotetext{
${ }^{11}$ Notamment pour le cancer du poumon et du côlon / rectum.

${ }^{12} \mathrm{http}: / /$ www.proinfoscancer.org/sites/default/files/2018-6-19-

la_vie_cinq_ans_apres_un_diagnostic_de_cancer_rapport_mel_inserm-inca.pdf
} 
alternatif et dynamique, adviennent comme un objet de négociations complexes. S'il est possible d'attacher un potentiel disruptif à l'expérience du cancer, celui-ci ne parvient pas à remettre en cause l'ordre du genre de manière frontale. Placé en dehors du seul cadre des activités du malade, entre projet de guérison et angoisse de rechute, le genre traverse l'expérience de la maladie dans ses composantes relationnelles, à la fois intimes et publiques (Meidani, Legrand et Jacques, 2015).

\section{En conclusion}


L'analyse met en évidence une interpénétration des questions de genre et de santé dans les expériences cancéreuses et les interactions de soins, examinées dans une perspective intersectionnelle et relationnelle. En effet, plus encore que les questions de sexe, c'est la perspective de genre qui éclaire sous un angle nouveau la socio-anthropologie du cancer.

Si le genre occupe une place si centrale dans la fabrique de l'expérience cancéreuse, c'est certainement aussi parce que l'âge médian du diagnostic se situe aux alentours de 61 ans pour le cancer du sein, et de 71 ans pour le cancer de la prostate. Dès lors, il n'est pas étonnant que les questions de genre priment sur les questions de travail ou d'âge, ni que les plus jeunes de nos enquêtés soient davantage présents dans les réajustements du genre qui prennent place suite à l'avènement du cancer - l'identité de genre et / ou la sexualité étant moins «figées »chez les plus jeunes de ces patient·es. Quant au niveau d'instruction et les appartenances classistes et ethno-raciales, ils se situent en deçà du genre, en ce sens que ce n'est pas n'importe quelle femme ou n'importe quel homme qui prend le pli des consignes médicales ou finit par s'en distancier, s'engage dans des pratiques préventives ou se contente des actions curatives élémentaires, intègre des protocoles expérimentaux, etc.

Tenant à distance le risque d'enfermer l'analyse dans l'opposition hommes-femmes, l'approche intersectionnelle permet de penser les différences à l'intérieur même du groupe des hommes et des femmes. Mais par-là même, elle rend plus difficile la distinction des aspects spécifiquement liés au genre. C'est pourquoi nous avons choisi de nous focaliser sur les cancers de la prostate et du sein. Le questionnement que nous portons, ainsi que nos conclusions, sont donc spécifiques à ces types particuliers de cancers. Laissant en retrait les populations atteintes des cancers du poumon et du côlon, notre analyse ne nous permet pas de généraliser nos résultats à une plus grande variété de situations cliniques, et c'est sans doute ici que se situe sa principale limite. On pourrait y entrevoir le signe d'une asthénie herméneutique, comme on pourrait y identifier ce qui lui confère toute sa richesse. Et toutes 
ces interrogations que le genre soulève sans forcément y répondre, en témoignent. D'autres travaux sont nécessaires afin de tester à plus large échelle les pistes interprétatives exposées dans cet article et étendre les résultats à d'autres localisations tumorales.

Si d'autres pistes de recherche restent à investiguer, cet article a permis d'étayer l'hypothèse selon laquelle les normes de genre concourent à définir l'expérience du cancer et l'expérience de soins. Plus encore, les données discutent un certain nombre de travaux qui appréhendent le genre comme le site par excellence de l'exercice de l'oppression, laissant de côté sa perception comme ressource. Ce faisant, l'analyse apporte un éclairage nouveau sur les potentiels effets positifs du genre sur le travail du malade et la dynamique interactionnelle.

Compte tenu du caractère épidémique des pathologies cancéreuses qui esquissent un problème de santé publique majeur, nous avons jugé judicieux d'engager une réflexion et contribuer à une compréhension des débats actuels autour du genre et de la maladie chronique. En accord avec cet objectif et en en faisant le point de départ de notre réflexion, le présent article adopte une posture inédite et montre, en quoi le concept du genre permet d'ouvrir le regard sur les expériences du cancer. Portée par un matériau ethnographique conséquent, la lecture proposée donne alors à voir le caractère opérationnel des concepts de l'identité et des technologies du genre et invite à leur usage. On ne saurait priver l'analyse de la maladie de son ancrage genré, relationnel, pluriel et intersectionnel, source d'inspiration et réalité observée par les chercheurs dont les travaux se portent à la lisière des expériences cancéreuses et des parcours de genre. Tout comme on ne pourra pas nier que le potentiel analytique d'une approche n'est pensable qu'à l'aune de ce qui lui fait obstacle et qu'il cherche à dépasser.

\section{Bibliographie}


ARRINGTON MI., 2004, « To Heal or not to Heal? On Prostate Cancer, PhysicianPatient Communication, and Sexuality », Journal of Loss and Trauma, 9, 2, p.159-166.

ABEL E.K., NELSON M.K., 1990, Circles of care: Work and identity in women's lives. Albany, NY: SUNY Press.

AHMED R., BATES B., 2007, «Gender Differences in Patients'Perceptions of Physicians'Cultural Competence in Health Care Interactions », Women's Health \& Urban Life, 2, 6, p. 58-80.

ALESSANDRIN A., ESTEVE-BELLEBEAU B., 2014, Genre !, Paris, Des ailes sur un tracteur.

AMSELLEM N., GAUTIER A., 2006, « Les représentations de la maladie et des soins », in GUIBERT P. et al. (dir.), Baromètre cancer 2005, Paris, INPES, p. 159- 178.

ARONSON J., 1992, «Women's sense of responsibility for the care of old people: "But who else is going to do it ?" », Gender \& Society, 6, 1, p. 8-29.

BAJOS N., BOZON M., 2008, Enquête sur la sexualité en France. Pratiques, genre et santé, Paris, La Découverte.

BATAILLE P., 2003, Un cancer et la vie. Les malades face à la maladie, Paris, Balland.

BAUER G.R., 2014, «Incorporating intersectionality theory into population health research methodology: Challenges and the potential to advance health equity », Social Science \& Medicine, 110, p. 10-17.

BELL K., 2010, «Cancer survivorship, mor(t)ality and lifestyle discourses on cancer prevention », Sociology of Health \& Illness, 32, 3, p. 349- 364.

BRAVERMAN L., 2018, «La mesure du PSA dans l'expérience du cancer de la prostate », in AMSELLEM N. (dir.), Le cancer : un regard sociologique. Biomédicalisation et parcours de soins, Paris, La Découverte, p. 13-27. 
BRUGÈRE F., 2014, Le sexe de la sollicitude, Lormont, Le Bord de l'eau.

BURY M., 1982, « Chronic illness as a biographical disruption », Sociology of Health \& Illness, 4, 2, p. 167- 182.

CANCIAN F., OLIKER S., 2000, Caring and gender, Thousand Oaks, CA: Pine Forge Press.

CARRICABURU D., MENORET M., 2004, Sociologie de la santé. Institutions, professions et maladies, Paris, Armand Colin.

CHRISTIAS P., 2009, « L'incorporation du moment du cancer dans l'histoire biographique des patientes atteintes d'un cancer chronique du sein (métastase, récidive, hormonothérapie adjuvante) », Sociétés, 3, 105, p. 71- 78.

COHEN P., SARRADON-ECK A.L., ROSSI I., SCHMITZ O., LEGRAND É., 2015, Cancer et pluralisme thérapeutique : enquête auprès des malades et des institutions médicales en France, Belgique et Suisse, Paris, L'Harmattan.

CONNELL R., 2014, Masculinités. Enjeux sociaux de l’hégémonie, Paris, Amsterdam.

COURTENAY W.H., 2000, «Constructions of masculinity and their influence on well-being: a theory of gender and health », Social Science \& Medicine, 50, 10, p. 13851401.

DEBREZ B., ROLLIN Z., 2016, Sociologie du cancer, Paris, La découverte.

DUBET F., 1995, Sociologie de l'expérience, Paris, Seuil.

DUFFY M., 2005, « Reproducing labor inequalities: Challenges for feminists conceptualizing care at the intersections of gender, race, and class », Gender \& Society, 19, 1, p. 66-82.

EAKER S. WIGERTZ A., LAMBERT P.C., BERGKVIST L., AHLGREN J., et al., 2011, « Breast cancer, sickness absence, income and marital status. A study on life situation 
1 year prior diagnosis compared to 3 and 5 years after diagnosis », PLoS One, 6, 3, http://e18040. doi:10.1371/journal. pone.0018040.

ESTEVE-BELLEBEAU B., 2018, «Cancer, corps et identité de genre », in A. MEIDANI et A. ALESSANDRIN (dir.), Parcours de santé. Parcours de genre, Presses Universitaires du Midi, Toulouse (sous presse).

FASSIN D., 2000, Les enjeux politiques de la santé. Études sénégalaises, équatoriennes et françaises, Paris, Karthala.

GALAND C., SALES- WUILLEMIN E., 2009, « Apports de l'étude des représentations sociales dans le domaine de la santé », Sociétés, 3, 105, p. 35- 44.

GERSTEL N., GALLAGHER S., 2001, « Men's caregiving: Gender and the contingent character of care », Gender \& Society, 15, 2, p. 197-217.

GLASER BG., STRAUSS A., 2010, La découverte de la théorie ancrée. Stratégies pour la recherche qualitative, Paris, Armand Colin.

GRECO C., 2016, « Shining a light on the grey zones of gender construction: breast surgery in France and Italy », Journal of Gender Studies, 25, 3, p. 303-17.

HENRY M., BAAS C., MATHELIN C., 2010, « Reconstruction mammaire après cancer du sein : les motifs du refus », Gynécologie Obstétrique, Fertilité, 38, 3, p. 217-223.

HERZLICH C., 1984, « Médecine moderne et quête de sens », in M. AUGE, C. HERZLICH (dir.), Le Sens du mal. Anthropologie, histoire et sociologie de la maladie, Paris, Éd. des Archives contemporaines.

HERZLICH C., PIERRET J., 1984, Malade d'hier, malade d'aujourd'hui, Paris, Payot.

JACQUES B., 2009, «Quitter l'identité de malade, le cas des femmes atteintes de cancer du sein », in COUSSON-GELIE F., LANGLOIS E., BARRAULT M. (dir), Faire Face au cancer. Image du corps, image de soi, Éd. Tikinagan. 
JAUNAIT A., CHAUVIN S., 2013, «Intersectionnalité », in BERENI A. (dir.), Dictionnaire genre et science politique, Presses de Sciences Politiques, p. 286-297.

KLEINMAN A., 1988, The Illness Narratives. Suffering, Healing and the Human Condition, New York (NY), Basic Books.

LAURETIS T. de, 2007, Théorie queer et cultures populaires, Paris, La Découverte. LEGRAND É., MEIDANI A., 2018, «Les femmes salariées face aux risques toxiques pour la reproduction : genre, santé reproductive et risques professionnels », in A. MEIDANI, A. ALESSANDRIN (dir.), Parcours de santé. Parcours de genre, Presses Universitaires du Midi, Toulouse (sous presse).

LORBER J., MOORE L.J., 2002, Gender and the Social Construction of Illness, $2^{\text {nd }}$ ed., New York, Altamira Press.

MASSÉ R., 1995, Culture et santé publique. Les contributions de l'anthropologie à la prévention et la promotion de la santé, Montréal, Gaëtan Morin.

MATHIESON C.M., STAM H.J., 1995, « Renegotiating identity: cancer narratives », Sociology of Health \& Illness, 17, 3, p. 283- 306.

MCCALL L., 2005, « The Complexity of Intersectionality », Journal of Women in Culture and Society, 30, 3, p. 1771-1800.

MCMAHON M., 1995, Engendering motherhood: Identity and self-transformation in women's lives, New York, The Guildford Press.

MEIDANI A., 2007, Cancers et genre : des enjeux de prévention à l'adhésion aux soins, Rapport I pour l'INCa.

MEIDANI A., 2008, «Le genre dans l'histoire de la maladie cancéreuse : le façonnement sociétal de la santé et ses inégalités », Sociologie Santé, 28, p. 81-120. 
MEIDANI A. 2018, «Le cancer a-t-il un genre ?», in A. MEIDANI, A. ALESSANDRIN, 2018, Parcours de santé, Parcours de genre, Toulouse, Presses Universitaires du Midi (sous presse).

MEIDANI A., ALESSANDRIN A., 2017, «Transidentités et cancers : l'émergence d'une nouvelle population à risques ?», Sciences sociales et santé, 35, 1, p. 41-64.

MEIDANI A., BOUSIGUE J.Y., 2019, Vivre la mort, Presses Universitaires du Midi (sous presse).

MEIDANI A., CAVALLI S., 2018, «Vivre le vieillir: autour du concept de déprise », N thématique, Revue Gérontologie \& Société, n¹55, vol. 40.

MEIDANI A., CAVAlli S., 2019, Figures du vieillir et formes de déprise, Toulouse, Érès.

MEIDANI A., GARNUNG M., 2009, La place des rapports sociaux de sexes dans les pathologies cancéreuses : une approche quantitative. Rapport III pour l'INCa.

MEIDANI A., LEGRAND É., JACQUES B. (dir.), 2015, La santé : du public à l'intime, Presses de l'École des Hautes Etudes en Santé Publique.

MÉNORET M., 1999, Les Temps du cancer, Paris, CNRS Éditions.

MENVIELLE G., CHASTANG J.F., LUCE D., LECLERC A., pour le groupe EDISC, 2007, «Évolution temporelle des inégalités sociales de mortalité en France entre 1968 et 1996. Étude en fonction du niveau d'études par causes de décès », Revue d'épidémiologie et santé publique, 55, 2, p. 97-105.

MESLÉ F., 2004, «Écart d'espérance de vie entre les sexes : les raisons du recul de l'avantage féminin », Revue d'Epidémiologie et de Santé Publique, 52, 4, p. 333-352.

MOCK V., 1998, «Breast cancer and fatigue: issues for the workplace », American Association of Occupational Health Nurses, 46, 9, p. 425- 431. 
MOLINIER P., 2013, Le travail du care, Paris, La Dispute, coll. Le genre du monde. MUZZIN L.J., ANDERSON N.J, FIGUEREDO A.T, GUDELIS S.O., 1994, « The experience of cancer », Social Science \& Medicine, 38, 9, p. 1201- 1208.

NUSSBAUM R., 2001, "Studies of women's health care », The Permanente Journal, 4, p. 62-67.

O'BRIEN R., HUNT K., HART G., 2007, « Standing out from the herd: men renegotiating masculinity in relation to their experience of illness », International Journal of Men's Health 6, 3, p. 178-200.

O'GRADY H., 2005, Woman's Relationship with Herself: Gender, Foucault and Therapy, London and New York, Routledge.

POTTS L. (dir.), 2000, Ideologies of Breast Cancer : Feminist Perspectives, London, Macmillan Press.

SAILlANT F., 1988, Cancer et culture. Produire le sens de la maladie, Montréal, Éd. Saint- Martin.

TARANTINI C., GALLARDO L., PERETTI-WATEL P., 2014, « Travailler après un cancer du sein. Enjeux, contraintes et perspectives », Sociologie, 2, 5, p. 139-155.

THÉBAUD-MONY A., 2008, «Construire la visibilité des cancers professionnels. Une enquête permanente en Seine-Saint-Denis », Revue française des affaires sociales, 2-3 (avril-septembre), p. 237-254.

THÉRY I., 2016, Mariage et filiation pour tous. Une métamorphose inachevée, Paris, Le Seuil.

TIEFER L., 1994, «The Medicalization of Sexuality », Annual Review of Sex Research, 7, p. 252-282.

TRONTO J., 1993, Moral Boundaries. A Political Argument For an Ethic of Care, New York - London, Routledge. 
VOEGTLI M., 2004, « Du jeu dans le Je : Ruptures biographiques et travail de mise en cohérence », Lien social et politique, 51, p. 145-158. 